\title{
MNEMOSINE: BIBLIOTECA DIGITAL DE LA OTRA EDAD DE PLATA (ORÍGENES, CONTENIDOS, PERSPECTIVAS) $^{1}$
}

\author{
MNEMOSYNE: A DIGITAL LIBRARY \\ OF THE OTHER SILVER AGE \\ (ORIGINS, CONTENTS, PERSPECTIVES)
}

\author{
José Miguel GONZÁLEZ SORIANO \\ Universidad Complutense de Madrid \\ josemigo@ucm.es
}

Resumen: Impulsada por los grupos de investigación LOEP e ILSA de la Universidad Complutense de Madrid, la Biblioteca Digital Mnemosine selecciona, cataloga y hace visibles en formato digital textos pertenecientes a un repertorio olvidado de la literatura española entre los años 1868 y 1936, permitiendo así la necesaria revisión historiográfica de este periodo. Junto al acceso a libros digitalizados y a la consulta de su base de datos, Mnemosine representa un campo de experimentación para el modelado de datos de colecciones literarias específicas y la creación de redes semánticas interoperables, generando investigación e innovación de calidad y nuevos modelos teóricos de interpretación de textos literarios.

Palabras clave: Biblioteca digital. Mnemosine. Otra Edad de Plata. Metadatos. Colecciones literarias. Innovación para la investigación.

Abstract: Powered by two research teams affiliated with the Complutense

\footnotetext{
${ }^{1}$ Esta publicación es parte de los resultados de investigación del Proyecto MNEMOSINE, "Hacia la Historia Digital de La Otra Edad de Plata: producción, almacenamiento, uso y difusión" (ref. RTI2018-095522-B-100), financiado por el Ministerio de Ciencia, Innovación y Universidades (MCIU), la Agencia Estatal de Investigación (AEI) y el Fondo Europeo de Desarrollo Regional (FEDER).
} 
University of Madrid, LOEP and ILSA, Mnemosyne Digital Library selects, catalogs and makes visible in digital format texts belonging to a forgotten repertoire of Spanish literature between 1868 and 1936, thus allowing the necessary historical reevaluation of this period. Together with access to digitized books and the consultation of its database, Mnemosyne is an experimental field for modeling data from specific literary collections as well as the creation of interoperable semantic webs, generating quality research and innovation and new models for the literary study.

Key Words: Digital library. Mnemosine. Other Silver Age. Metadata. Literary Collections. Innovation for research.

\section{PRESENTACIÓN}

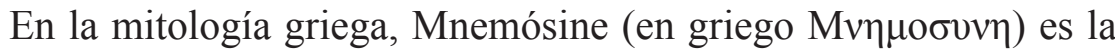
personificación de la memoria y madre — con Zeus - de las nueve musas. La mnemotecnia, término derivado de la raíz mnémē ('memoria'), se define como aquellas técnicas para el almacenamiento de conocimientos. Partiendo de datos empíricos estructurados y de textos digitalizados como almacén literario, la Biblioteca Digital Mnemosine pretende llevar a cabo una revisión historiográfica de la conocida como Edad de Plata de la literatura española, mediante herramientas digitales que permitan a los investigadores el conocimiento de la época a través de nuevas colecciones, las cuales harán visibles nuevos subgéneros, ejes temáticos, diversidad de lectores y monográficos de autores hasta configurar, con perfiles propios, lo que ya se viene denominando "La Otra Edad de Plata" (Ena Bordonada, 2013: 13).

Mnemosine es una biblioteca digital de acceso abierto que comprende textos literarios "raros y olvidados" de nuestra literatura producidos entre 1868 y 1936 y relegados durante décadas a los márgenes de un canon muchas veces discriminatorio (Romero López, 2014). Tratándose de una época de gran brillantez en las letras españolas, con frecuencia ha quedado injustamente reducida, a la hora de establecer el canon correspondiente, a una serie de nombres y de obras que responde tan 
solo a aquellos catalogados como primeras figuras; sin embargo, conviene no olvidar que tales escritores surgieron en el seno de un vasto e influyente contexto, enriquecido por la pluralidad y convivencia de diversas personalidades, tendencias, estilos y temas, hasta conformar un conjunto de manifestaciones cuya riqueza cultural y literaria debe recuperarse dentro de la reestructuración que la historiografía del periodo necesita (González Soriano, 2017: 12).

El término "raros" aplicado de forma crítica a la literatura española apareció por vez primera en 1863, en el Ensayo de una biblioteca española de libros raros y curiosos de Bartolomé José Gallardo, obra que, junto al Nuevo ensayo... publicado un siglo después por Homero Serís (1964), mostraba la preocupación del racionalismo ilustrado por recabar para la historia de la literatura a todos los escritores, no solamente los consagrados (Romero López, 2014: 412). El doblete "raros y curiosos" hizo fortuna, pero el segundo término daría paso a los "olvidados" a partir de la refundación terminológica establecida por Federico Carlos Sainz de Robles (1898-1983), estudioso de la Edad de Plata que iniciaría en 1971 la recuperación historiográfica de este periodo tras publicar Raros y olvidados (la promoción de "El Cuento Semanal"), coincidiendo con una tímida apertura liberalizadora en el llamado tardofranquismo toda vez que la censura del régimen había reducido drásticamente, durante décadas, la nómina de autores y temas existente antes de la Guerra Civil. En su libro, Robles intentaría una valoración generacional de la época al hablar de "olvidados" no tanto en un sentido de escasa circulación o impacto, sino en referencia a aquellos escritores que se caracterizaron por una estética o por una ideología sin encaje dentro de los patrones críticos establecidos. Así, sus nombres se vieron suprimidos de la cultura oficial del franquismo a causa de su adscripción política - lo que conllevaba también, en la mayoría de los casos, la condena impuesta por la crítica académica- o por haber cultivado un tipo de literatura no considerada de vanguardia en su época — aunque gozase del favor del público y fuera entonces muy leída ${ }^{2}$ - Pero en aquella exclusión del canon hubo igualmente otros motivos: biológicos (muerte prematura del autor, nacimiento en el extranjero...), genéricos (ser mujer y literata), de accesibilidad (al haber desarrollado el grueso de su producción en la prensa, medio de carácter efímero en sí mismo), etc.

${ }^{2}$ Véase al respecto el esclarecedor volumen colectivo editado por Ceballos Viro (2014). 
Among the motives behind the exclusion of particular authors from the Silver Age canon are factors of a biographical, ideological, and generic nature. Some authors died young or were born outside of Spain. Other writers were killed, died in prison, went into exile (forced or voluntary), or were otherwise censured and silenced following the Civil War. These authors remained marginal to the canon created by critics in Spain under the Franco regime. Additionally, women writers and those concerned with queer sexualities often met with dismissal. Exclusion was also possible on aesthetic grounds and even on account of the design and physical format of particular texts (Romero, Bueren y Gayoso, 2017: 401).

Dentro de la Biblioteca Digital Mnemosine, se habla de textos raros u olvidados y no tanto de autores, al ser contemplada la literatura en sí y no tan solo los literatos; lo que permite - por ejemplo — catalogar la literatura escrita por mujeres como categoría paralela a la escrita por hombres, pero con singularidades. Una de las dificultades más habituales que nos encontramos al realizar estudios "de género" sobre la actividad intelectual femenina - tan en boga en la actualidad — es, precisamente, que las bases de datos de las bibliotecas con fondos digitalizados no discriminan los géneros de los autores; Mnemosine, en cambio, pretende que los yoes de estas autoras sean visibles y que la investigación los tenga en cuenta. Esta organización textual de la biblioteca permite asimismo la interliterariedad (traducciones, redes de contacto, etc.) y la transdisciplinariedad o vinculación entre la literatura y el arte, la ciencia, el cine, la música...

Mnemosine, Biblioteca Digital de La Otra Edad de Plata (18681936) abarca un periodo comprendido entre dos fechas cruciales de la Modernidad histórica española: el año de 1868 constituye el punto de partida ya que, a partir del Sexenio democrático, se desarrolla en España la novela realista, la poesía posromántica, modernista y simbolista, el teatro burgués y la zarzuela; la fecha de 1936, inicio de la Guerra Civil, cercena las tendencias literarias modernas debido a la muerte, silencio o exilio de numerosos escritores (Romero López, 2014: 411; Romero, Bueren y Gayoso, 2017: 399). Con un criterio de similar amplitud, extendiendo su comienzo hasta 1868 frente al más habitual de 1898, el año del "desastre", el término Edad de Plata — que parte en su génesis de José Manuel Blecua - sería utilizado por Miguel Martínez Cuadrado en su contribución a la Historia de España, dirigida por él y por Miguel Artola 
(La burguesía conservadora, 1868-1931) publicada en Alianza en 1973; dos años anterior a la emblemática obra de José-Carlos Mainer La Edad de Plata (1902-1939). Ensayo de interpretación de un proceso cultural que populariza el término, englobando su estudio entre 1902, fecha de la aparición de las cuatro grandes novelas que marcan el inicio de una nueva narrativa en España (La voluntad de Azorín; Camino de perfección de Pío Baroja; Amor y pedagogía de Unamuno y Sonata de otoño de ValleInclán), hasta los años de la Guerra Civil, que incorporaría en su segunda edición de 1980.

Ya en 2013, aparecerá el volumen colectivo editado por Ángela Ena Bordonada La Otra Edad de Plata. Temas, géneros y creadores (18981936), otorgando carta de naturaleza a este término o concepto de "otredad" para aquella producción literaria de la época "que ha permanecido y permanece entre las sombras" (Ena Bordonada, 2013: 13), como fruto del primer seminario llevado a cabo en noviembre de 2010 por el grupo de investigación Temas y Géneros de la Edad de Plata (TGEP) de la Facultad de Filología de la Universidad Complutense de Madrid, hoy llamado La Otra Edad de Plata: Proyección Cultural y Legado Digital (LOEP); y en donde - como se dijo entonces - se oyeron nombres de escritores, títulos de obras y modalidades literarias que la mayoría del público desconocía, lo que supuso una de nuestras grandes compensaciones. Este grupo de investigación LOEP, junto al grupo de la Facultad de Informática ILSA (Implementation of Language-Driven Software and Applications) de la misma Universidad, serán los impulsores de la creación, poco después, de Mnemosine, Biblioteca Digital de La Otra Edad de Plata, en el marco del Proyecto de Investigación I+D "Escritorios electrónicos para las Literaturas-2" y que aborda el conocimiento de este periodo históricocultural configurándose a un tiempo en herramienta y plataforma objeto de estudio en sí misma: constituye - como se ha mencionado - tanto una base de datos y repertorio de textos digitalizados de acceso abierto que permite la revisión historiográfica especializada y crítica de la literatura de la Edad de Plata, como además, en tanto que laboratorio experimental de modelado de datos, un espacio de creación de propuestas metodológicas concretas (Cotarelo Esteban, 2018: 26). 


\section{GESTACIÓN Y DISEÑO DE UNA SMARTLIBRARY}

Con fecha de 5 de septiembre de 2012, la subdirección general del Ministerio de Economía y Competitividad concedía a varios de los miembros integrados en los grupos de investigación LEETHI (Literaturas Española y Europeas del Texto a la Hipermedia), y el actual LOEP de la Universidad Complutense financiación oficial para el proyecto "Escritorios electrónicos para las Literaturas-2" (Ref. FFI2012-34666), lo que significaba el punto de arranque en la creación de una nueva biblioteca digital de textos "raros y olvidados" de la Edad de Plata, llamada Mnemosine, ideada y coordinada por Dolores Romero López, profesora titular de la Facultad de Filología de la UCM. Con la entrada del siglo XXI y la nueva era tecnológica, habían dado comienzo los grandes procesos de digitalización masiva de libros antiguos y modernos, propiciando una renovación teórica y de metodología fundamental que aportaba novedosos enfoques (lectura distante, el uso de las TIC, etc.) en los estudios filológicos, la investigación y la docencia. El acceso a los fondos de las bibliotecas y hemerotecas nacionales y especializadas a través de Internet es hoy ágil e inmediato; y en nuestro caso, podemos ya leer con gran facilidad textos de autores de la Edad de Plata que habían sido considerados menores o secundarios por la historiografía moderna. Su actual disponibilidad amplía y modifica el conocimiento previo que teníamos de la época, dando lugar a nuevos planteamientos críticos pues, una vez pasada — en buena medida - la fase inicial de digitalización masiva de fondos por parte de las instituciones, todo ese vasto conjunto de documentos, publicaciones, recursos u objetos virtuales precisa de ser organizado para poder inferir nuevos conocimientos que generen investigación e innovación de calidad. A las grandes bibliotecas digitales como la Biblioteca Digital Hispánica de la Biblioteca Nacional de España, impulsada en 2008 y cuyo crecimiento sigue siendo constante, les interesa y resulta fundamental, sin embargo, la estructuración, difusión y optimización de todo el material ya digitalizado, para lo cual han de recabar el concurso de especialistas; un hecho que, en nuestra disciplina, abre nuevas perspectivas para los filólogos ${ }^{3}$ al constituir

\footnotetext{
${ }^{3}$ Tal fue el título y leitmotiv de la XXVII edición de uno de los Cursos de verano de la UNED celebrado del 27 al 29 de junio de 2016 en el Centro Asociado de Ponferrada, Introducción a las Humanidades Digitales: nuevas oportunidades y perspectivas para los filólogos, dirigido por Elena Bárcena y coordinado por María Goicoechea de Jorge, donde se analizaron estas
} 
esa, sin duda, una de sus tareas primordiales dentro del campo de las Humanidades Digitales: organizar, clasificar, implementar datos, modelar nuevas colecciones... Se precisa para esa reutilización específica, y están surgiendo cada vez más, pequeñas bibliotecas digitales desarrollando métodos para aumentar la eficacia en la transmisión de los documentos por la red.

La primera plataforma de Mnemosine, en versión beta, se localizaría en los servidores de la Biblioteca de la Universidad Complutense de Madrid, como resulta lógico al haber surgido en el seno de dicha institución ${ }^{4}$. Su andadura principiaría así a finales de 2012, tras la importación masiva de un amplio repertorio digital de autores y obras - previamente seleccionados por el grupo de investigación LOEP - procedentes de dos repositorios principales: HathiTrust Digital Library, promovida por Google Books, una asociación conformada por 66 - actualmente más de 80 - de las principales bibliotecas académicas y de investigación —incluida la Library of Congress o la Biblioteca de Oxford - de la cual la Complutense se convirtió en socia en noviembre de $2010^{5}$, y cuyo software permite crear colecciones particulares por parte de usuarios; y la Biblioteca Digital Hispánica, con la que se materializó un protocolo de entendimiento en la primavera de 2012, con el fin de digitalizar libros pertenecientes a autores "raros y olvidados" de la Edad de Plata que la Biblioteca Nacional poseía entre sus fondos. Desde HathiTrust se localizaron un total de 2.873 ítems correspondientes a libros publicados —o con participación secundaria en los mismos - por los autores señalados para su inclusión en Mnemosine — si bien tan solo 433 con acceso a "vista plena" (full view) - ; y del acercamiento inicial a $B D H$ se estimaron unos fondos de hasta 2.448 obras de autores "raros y olvidados" a digitalizar y otros 1.017 títulos de autoría femenina (Romero López, 2014: 418; Romero, Bueren y Gayoso, 2017: 405).

Inicialmente alojada nuestra biblioteca en el repositorio $O d A$, una

cuestiones y tuvimos ocasión de participar, junto a diversos especialistas en edición electrónica y la digitalización de fondos bibliográficos, con la ponencia "Mnemosine, Biblioteca Digital de La Otra Edad de Plata".

${ }^{4}$ Dirección web: http://repositorios.fdi.ucm.es/mnemosine/ [12/05/2029].

${ }^{5}$ Desde la página web de la Biblioteca de la UCM existe un enlace de acceso a HathiTrust: https:// biblioteca.ucm.es/hathitrust/ [12/06/2019]. 
RIA (Rich Internet Application) de código abierto 6 que tiene como finalidad la creación y gestión de colecciones digitales, no obstante Mnemosine se trasladó poco después a Clavy — ambas aplicaciones desarrolladas por el grupo de investigación ILSA - para poder importar e integrar los objetos digitales de las dos bibliotecas mencionadas con anterioridad:

OdA se usa para crear de forma inductiva colecciones de objetos digitales en dominios altamente especializados. Es una herramienta orientada a usuarios no necesariamente especialistas en informática, que necesitan crear de forma sencilla y dinámica colecciones de objetos digitales con esquemas de datos totalmente ajustados a su dominio de conocimiento, a sus necesidades y a sus múltiples formas, posiblemente diferentes a lo largo del tiempo, de entender, describir y organizar los objetos de sus colecciones.

[...] Clavy tiene las mismas capacidades de reconfiguración y creación incremental de colecciones que OdA y, además, aporta mecanismos de manejo de múltiples colecciones en un mismo repositorio, mecanismos para la importación y exportación de objetos digitales desde y hacia otros repositorios $y$, de forma novedosa, mecanismos para transformar y unificar esquemas de metadatos de colecciones diferentes facilitando al máximo la integración de repositorios y la importación y exportación (FernándezPampillón, Gayoso, Sarasa y Sierra, 2018: 75-76).

Efectuada esta primera importación masiva de títulos y autores a través de Clavy, el inmediato paso posterior consistió en una labor de content curation (cotejo, limpieza, borrado...) a cargo de un curator ("curador"), gestor bibliotecario y filólogo especialista en contenidos en nuestro caso, de los aproximadamente 4.000 objetos digitalizados procedentes de HathiTrust y de $B D H$. Así, en primer lugar, se eliminaron todos aquellos elementos repetidos (autores/autoridades y ediciones de obras) al estar presentes simultáneamente en una y otra biblioteca o se corrigieron de forma manual determinados caracteres o grafías erróneas en la traslación automática de un idioma a otro — del inglés al españoldada su especificidad, como la letra $\tilde{n}$ o las tildes. Seguidamente, para la generación del modelo de datos de Mnemosine se procedió a la adaptación y enriquecimiento de los datos importados, depurando y detallando al máximo los contenidos y proponiendo otros nuevos. En esta fase se llevaría

${ }^{6}$ OdA se puede descargar de https://github.com/ILSA-UCM/OdA [12/06/2019]. 
a cabo el mapeo entre los elementos de MARC 21 —el formato más usado aún en los actuales sistemas de automatización de bibliotecas - y los del modelo de datos definido para Mnemosine (Figura 1).

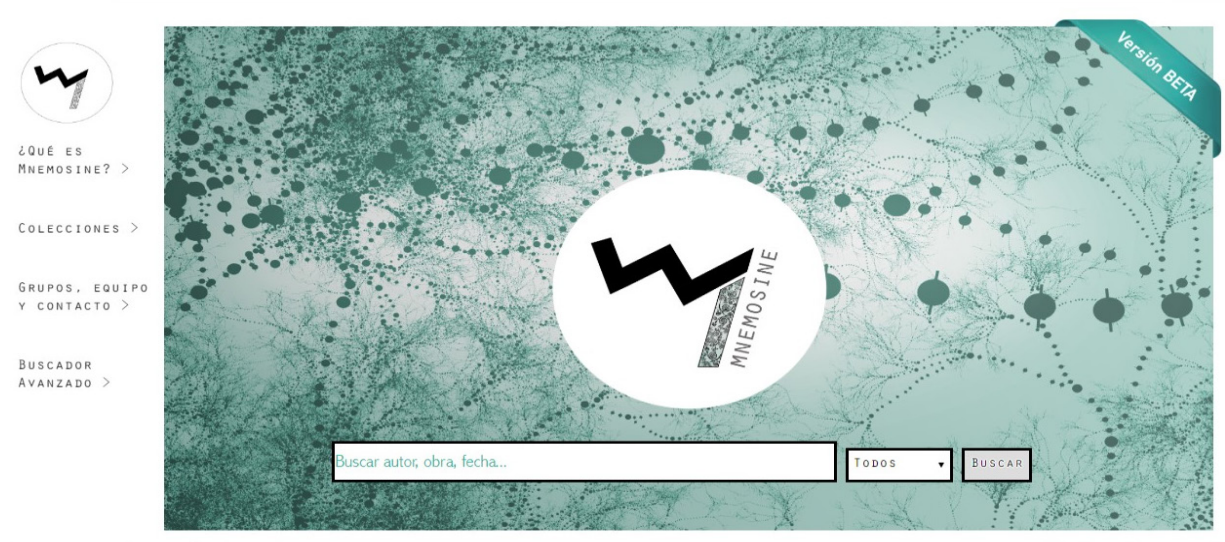

Figura 1. Diseño gráfico de Mnemosine (2015-2019)

Clavy posibilita la importación, exportación y edición de registros desde múltiples formatos como MARC 21, en el que se hallaban codificados los metadatos de los objetos digitalizados procedentes de Hathitrust y de $B D H$ siguiendo las normas bibliotecarias habituales de catalogación — fijadas por la $I S B D$ —, además de su integración en un modelo prediseñado de Mnemosine para su exportación posterior a otros formatos hipertextuales compatibles, como HTML o xml, u otros sistemas como OdA (Fernández-Pampillón, Gayoso, Sarasa y Sierra, 2018: 7677). Como resulta natural, en algunas ocasiones el modelo de datos de Mnemosine no ha requerido el nivel de detalle presente en MARC 21; y en otras, ha sido necesario incorporar metadatos que no se hallan en dicho formato, que no distingue - por ejemplo - entre un autor hombre/mujer ni especifica su nacionalidad, al no necesitarlo en su diseño bibliotecario funcional de búsqueda y recuperación, que no tiene por qué coincidir con las necesidades del mundo de la investigación y la docencia. A la hora de fijar el modelo de datos para Mnemosine, por otra parte, se establecieron como principios generales que aquel debía de ser extensible, permitiendo incorporar nuevos elementos de descripción; estar estructurado, siendo XML un estándar de facto para el intercambio de información; mostrarse en lo posible normalizado, fomentando el empleo de estándares en todo el 
proceso para asegurar la interoperabilidad de $\operatorname{los}_{\text {datos }}^{7}$; y su diseño debía ser compatible con los principios de web semántica de interacción entre un usuario y un agente de software (Codina y Rovira, 2006: 17) y de datos enlazados, lo cual no habría de resultar complejo pues dicha web parte de un modelo conceptual sencillo basado en entidades y relaciones, y el empleo de URLs y de lenguaje $R D F$ (con su estructura sujeto-predicadoobjeto) no constituye ningún obstáculo a la hora de expresar la información como sea necesario (Romero, Bueren y Gayoso, 2017: 406-407).

En este modelado de datos, son varios los elementos presentes en Mnemosine que no forman parte de la descripción bibliotecaria: la función del autor (o autoridad) con respecto a una obra, cuando es secundario; su género o identidad sexual; el lugar de nacimiento y muerte, de residencia y su geolocalización espacial; el uso de distintos seudónimos; la actividad o actividades profesionales; los géneros literarios cultivados y las influencias artísticas... Al incorporarlos, la finalidad es poder ofrecer al usuario una ficha-autor o una ficha-obra "enciclopédica" y, por tanto, especializada, aprovechando la extensibilidad de modelado que permite la aplicación Clavy. Pero a la vez, los datasets resultantes de los metadatos de Mnemosine se han de poder exportar a otras colecciones digitales, permitiendo así la fluencia masiva de datos. El resultado último será la consecución de una smartlibray (Romero López, 2014: 419), una biblioteca digital para la docencia y la investigación en la que se puedan implementar aplicaciones de uso personal o social y con avanzado nivel de conectividad, favoreciendo la búsqueda y recuperación de la información. La implementación de todo este proceso parte de un conocimiento previo por parte del especialista, del filólogo, sin el cual nada es posible: es necesaria su contextualización histórico-literaria-cultural.

\section{GRAMÁTICA Y OBJETOS DIGITALES DESDE $C L A V Y$}

Otra de las tareas fundamentales en la fase inicial de configuración

\footnotetext{
${ }^{7}$ Así, "the aim of thee data import process is not simply to save the time it would otherwise take to type the relevant bibliographic data. Starting with information from the catalogs of institutional libraries helps normalize the entry of data into particular fields, especially in the case of authors' names. Many libraries deploy systems of authority control to help standardize names and identify authors more accurately" (Romero, Bueren y Gayoso, 2017: 406).
} 
de la Biblioteca Digital Mnemosine - y en cualquiera de las fases posteriores - consistió en enlazar los objetos virtuales importados que también se pueden crear directamente- y divididos en dos clases o categorías fundamentales, "persona" y "obra" (Figura 2), entre sí; de modo que la primera tenga enlazados como recursos todas aquellas obras que correspondan a cada objeto virtual persona $\mathrm{y}$, en correspondencia biunívoca, los objetos virtuales obras tengan enlazados como recursos todos aquellos objetos virtuales de persona que le correspondan, en un tipo de relación (autor, traductor, ilustrador... o "escrita por", "traducida por", "ilustrada por") establecida gramaticalmente desde Clavy para dar cabida a la descripción más precisa de sus objetos (cada uno de ellos con un número ID de identificación automáticamente asignado) y colecciones. Esta estructura de modelo de datos enlazados permitirá con posterioridad realizar búsquedas complejas de datos cruzados.

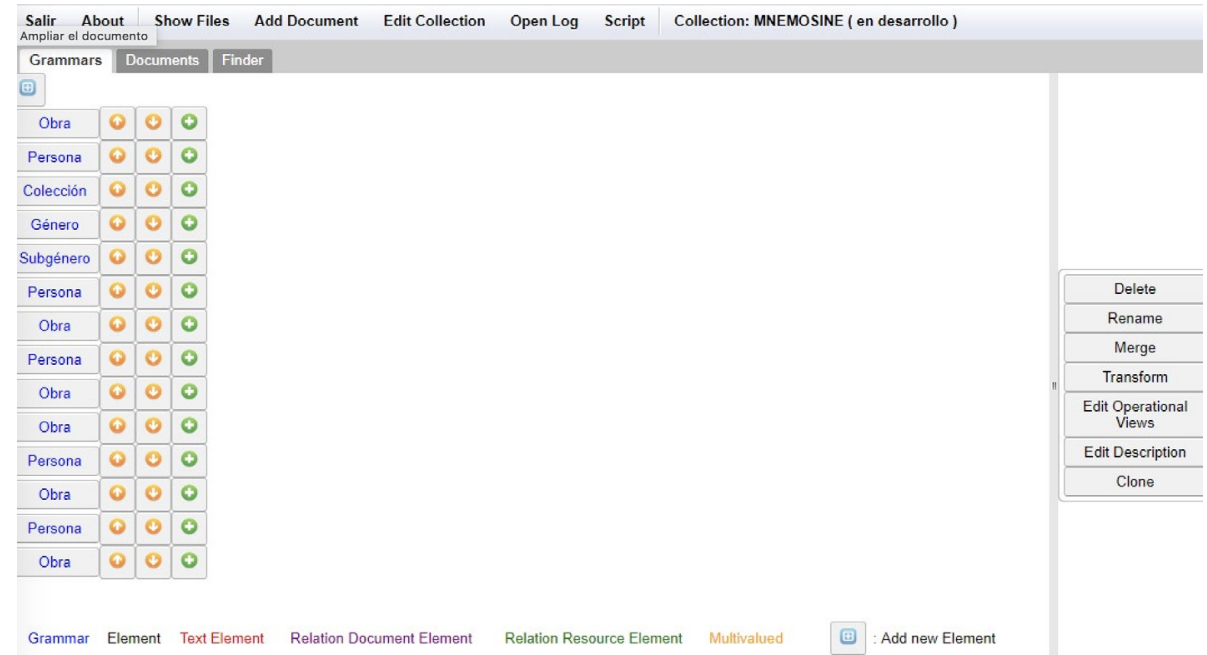

Figura 2. Visualización de la gramática de la herramienta Clavy.

Desde la página de inicio de Mnemosine, un hipervínculo permite el acceso a la herramienta informática Clavy, donde se importan todos los metadatos que ofrece la biblioteca y se crean otros nuevos ${ }^{8}$. Una vez dentro de esta aplicación, que constituye el armazón interno de Mnemosine,

${ }^{8}$ Dirección web: http://clavy.fdi.ucm.es/Clavy/ [12/06/2019]. 
para empezar a trabajar en él el administrador del sistema debe dar de alta a los futuros usuarios/autores de la colección, asignándoles los roles de creador de objetos digitales o de administrador, junto a permisos de escritura y edición sobre la colección o sobre objetos virtuales concretos. Dentro de la aplicación, además de una barra de tareas existen tres secciones diferenciadas en pestañas: Grammars, para la edición gráfica del esquema de metadatos de cada colección; Documents, para la edición de los objetos digitales y Finder para una búsqueda simple de esos objetos digitales o virtuales y de sus datos: valores que contienen los atributos o, sencillamente, palabras y texto que estén contenidos en dichos atributos (Fernández-Pampillón, Gayoso, Sarasa y Sierra, 2018: 77-78).

Dado que no es posible tener un único conjunto de metadatos que resulte eficaz para hacer una descripción de todos los objetos depositados en Mnemosine y - menos aún- que coincida con otros repositorios bibliográficos en red, nuestra biblioteca utiliza, y su uso está muy extendido en la actualidad, el lenguaje RDF (Resource Description Framework) que permite la codificación, el intercambio y la reutilización de metadatos estructurados y hace posible la interoperabilidad de los mismos, mediante convenciones sintácticas y semánticas basadas en XML. RDF suministra un modelo que permite utilizar cualquier vocabulario que se necesite para definir los elementos (clases, en terminología RDF) que se van a considerar, y las relaciones (propiedades) entre dichos elementos. Las propiedades asociadas y que expresan esas relaciones se denominan tipos de propiedad; y a los tipos de propiedad les corresponden valores, llamándose descripción a una colección de propiedades referidas al mismo elemento. Si los enunciados "El autor [propiedad] del Doc1 es Carmen de Burgos" y "Carmen de Burgos [elemento 1] es el autor del Doc1 [elemento 2]" expresan lo mismo, para una máquina son dos cadenas completamente distintas (Figura 3). Usando un modelo de datos con tres tipos de componentes (elementos, tipos de propiedad o de relaciones y los valores correspondientes), RDF da un método no ambiguo de expresar la semántica en una codificación legible por la máquina (García Camarero y García Melero, 2001: 170-174). Para el desarrollo de Mnemosine y sus colecciones es posible, por tanto, crear una ontología única y singular; sin embargo, siempre será preferible, para la creación de esa ontología, reutilizar vocabularios ya existentes o normalizados y evitar un número elevado de elementos propios, favoreciendo así que el resto de sistemas 
comprenda nuestro modelo de datos y puedan reutilizar a su vez la información contenida9 .

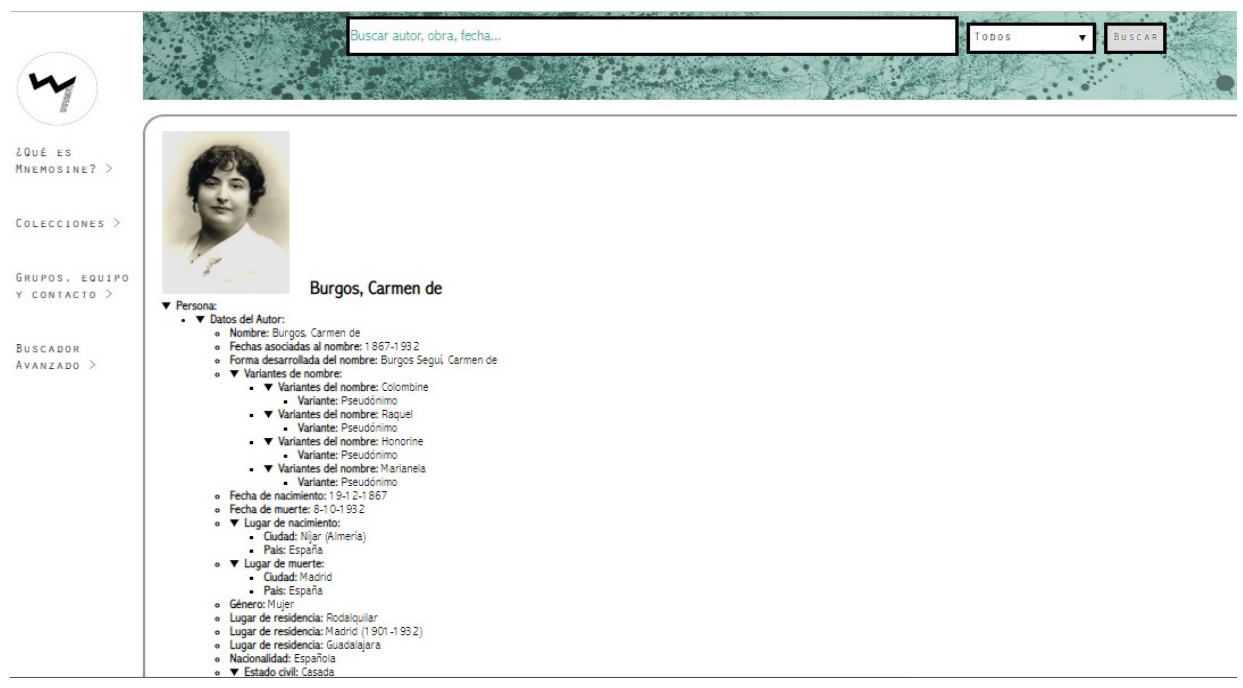

Figura 3. Ficha de autor en Mnemosine.

En la fase de creación del esquema de metadatos dentro de Clavy, el curator o especialista en el dominio de la colección empieza creando los atributos y subatributos y valores desde la pestaña Grammars. Cada atributo dentro de un esquema puede ser de los siguientes tipos: estructura (no permiten valores asociados, solo estructuran los atributos que contienen), de texto (asociar valores de texto a los objetos virtuales que posean implementaciones de estos atributos), de relación (para crear relaciones entre objetos virtuales dentro de una misma colección) y de recurso (permiten asociar recursos a un objeto: tanto externos a través de su $U R L$, como internos asociados a la colección y depositados dentro de Clavy). Las gramáticas tienen una estructura de árbol y sirven de esquema de orden a los valores de los objetos digitales. Además, cada atributo podrá ser único o multievaluado en función de si es preciso o no que pueda reduplicarse (así,

\footnotetext{
9 "Some preexisting vocabularies that are useful for the definition of the Mnemosine ontology are Web Ontology Language (OWL), Functional Requirements for Bibliographic Records (FRBR), Friend of a Friend (FOAF) and Dublin Core. In addition to saving time, using preexisting vocabularies makes it easier to recycle information from the Mnemosine database" (Romero, Bueren y Gayoso, 2017: 406).
} 
para consignar varios autores — principales y secundarios - de una misma obra; o diferentes géneros cultivados por un autor; o diversos seudónimos o lugares de residencia del mismo...), permitiendo que un objeto digital tenga repeticiones de un atributo; y también podrá ser navegable, si se desea que aparezca en el menú de navegación de la colección, lo que los convertirá asimismo en indexados y en controlados. Adicionalmente, cada atributo puede tener asociado otro tipo de información que utilizarán internamente los conectores de importación y exportación (FernándezPampillón, Gayoso, Sarasa y Sierra, 2018: 77).

Una vez creado un esquema inicial de metadatos mínimo, el especialista puede crear los objetos virtuales en la pestaña Documents. En esta pestaña se muestra el menú de navegación — creado con los atributos seleccionados como navegables en la fase anterior-y los objetos digitales. Desde la pestaña Add Document situada en la barra de tareas inmediatamente superior, se abre un cuadro de diálogo donde crear un nuevo objeto virtual, estableciendo la clase correspondiente para el mismo. Los objetos digitales son un conjunto de pares atributo $<>$ valor de manera que solo existirá una relación a un valor por cada atributo. Si el atributo es multievaluado, se realizará un proceso interno de duplicado del árbol desde la estructura para mantener esta norma. Inicialmente, Clavy recoge para Mnemosine dos clases principales (personas y obras), entre las cuales establece diferentes relaciones: autor de / escrita por; traductor de / traducida por; compositor de / musicada por —en el caso de obras del teatro lírico-; etc. En el futuro, será necesario ir ampliando el número de clases - y de relaciones - para dar cabida a la descripción de sus nuevas colecciones, como así ha sucedido ya en alguna ocasión; por ejemplo, al establecer una clase colección para determinados objetos virtuales de títulos de colecciones de novela corta — tan populares durante el primer tercio del siglo XX, cuya irrupción fulgurante en el panorama editor y cultural supuso una auténtica revolución que transformaría la producción literaria de los escritores-donde poder insertar información general sobre las mismas mediante metadatos específicos; y enlazar en su interior como recursos aquellas obras que formaron parte de su catálogo y viceversa: enlazar en cada uno de los objetos virtuales de obras, además de su autor, la colección de novela corta a la que pertenecían. También, a la hora de conformar la colección de traductoras, se definió un nuevo recurso multievaluado, "reediciones", para asociar al objeto virtual de la 
obra traducida las reediciones posteriores a la edición original, que varían de fecha e incluso de editorial; y así poder seguir el recorrido filológico e histórico de aquella traducción (Figura 4).

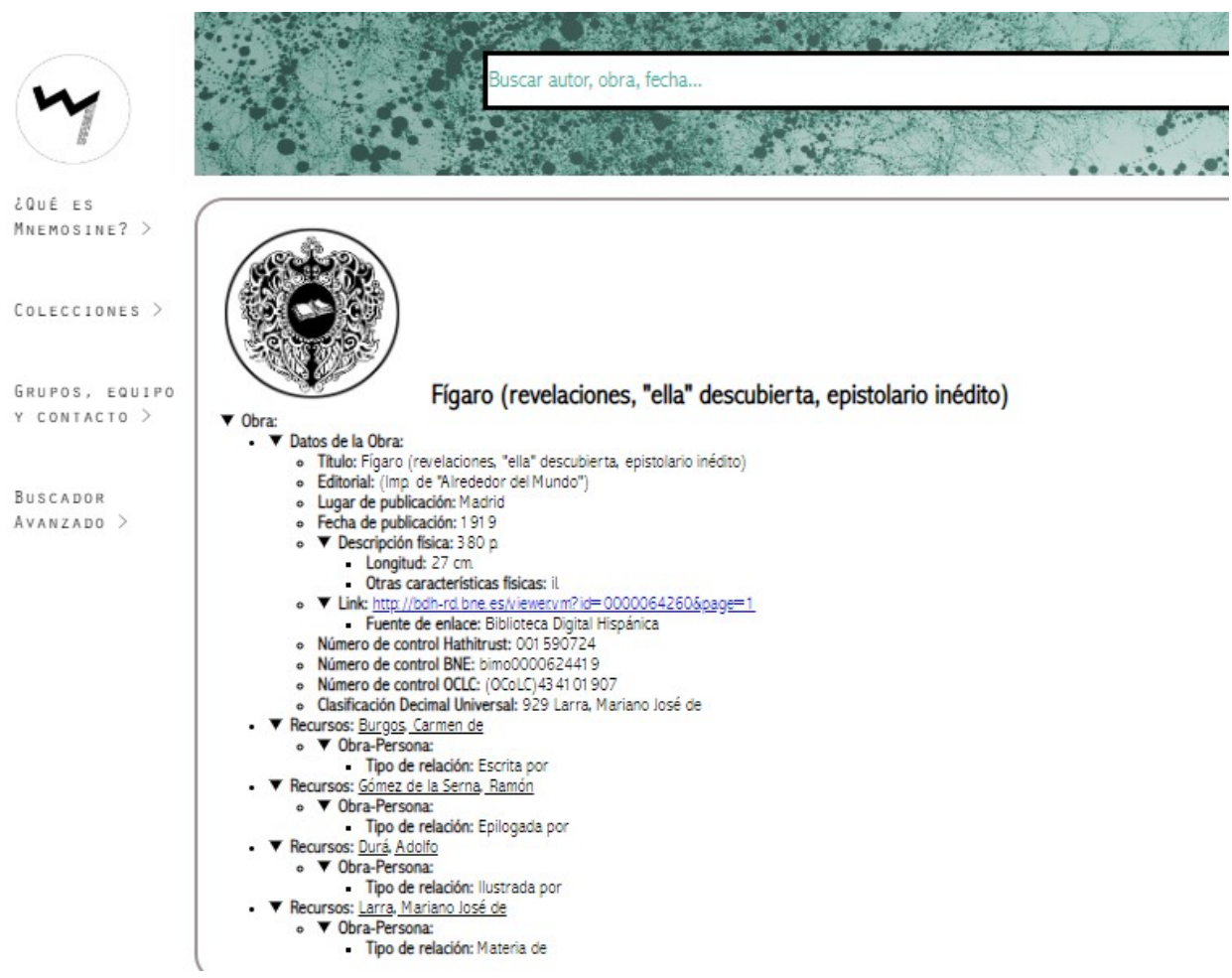

Figura 4. Ficha de obra en Mnemosine.

\section{LAS COLECCIONES LITERARIAS EN MNEMOSINE}

Finalizado este primer ciclo de búsqueda de textos ya digitalizados con los que nutrir Mnemosine, e inventariado ya como repositorio electrónico un corpus amplio de autores y obras asociados entre sí, el acceso a la base de datos se abre en 2015 una vez que el volumen de información acumulado se considera científicamente relevante, aun cuando con un número de libros con un enlace de acceso libre a su lectura relativamente modesto todavía. Así, en el marco del Curso de Verano de El Escorial Hacia bibliotecas digitales inteligentes para la docencia y la investigación, dirigido por las profesoras Dolores Romero y Amelia Sanz 


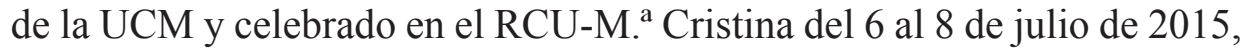
se presentó el diseño del interfaz de la web a través del cual se visualiza actualmente Mnemosine, a cargo de Laura Sánchez Gómez; y junto a los contenidos ya existentes, se habló de crear futuras colecciones temáticas y de autores dentro de la biblioteca, definiendo los adecuados motores de búsqueda avanzada y modelando los metadatos de acuerdo a los criterios y necesidades científicas de cada colección, si bien bajo los criterios - ya comentados - de operabilidad e intercambio de datos (Zhang, 2008) que permitan su hipotética exportación a otras colecciones digitales.

Los proyectos especializados dan valor al contenido y generan un nuevo modelado de datos abiertos enlazados. De ese modo se logrará un avance en el conocimiento de metadatos como género, lugares, funciones... Partiendo de un repertorio cada vez más ampliado, Mnemosine, Biblioteca Digital de La Otra Edad de Plata se estructura en colecciones particulares realizadas por especialistas como fruto de su propia investigación y del desarrollo tecnológico de la plataforma, a la hora de poder gestionar búsquedas cruzadas. Así, nuestra biblioteca cuenta en la actualidad con una docena de colecciones que hacen visibles diversos géneros, macrogéneros o subgéneros (Colección de Protociencia-Ficción, Diálogo Literario, Literatura Infantil), ejes temáticos novedosos (Madrid en la Literatura, Exilio Norteamericano), variedad de lectores (Literatura de Quiosco, Libros Interactivos), voces colectivas (Mujeres Intelectuales, Muertos en Conflicto (1936-1939), Traductoras de la Edad de Plata) y monográficos de autores (Colección Carmen de Burgos). Estas colecciones se organizan a su vez mediante un número variable de entidades de dos clases fundamentales: "persona" y "obra". Estas dos clases se interrelacionan, como vimos, estableciendo propiedades semánticas a través de aspectos tales como la autoría, la traducción o la ilustración, entre otros. La entidad o elemento "obra" se divide asimismo en dos ejes: "Datos de la obra" — características externas y de edición relativas al volumen-y "Contenido de la obra".

El modelado de datos permite adaptar los metadatos a la naturaleza diversa de las colecciones, en cuanto a su catalogación específica y a la futura posibilidad de una búsqueda afinada: todas ellas responden a algunos metadatos comunes, de descripción bibliográfica esencial muchos de ellos y también específicos de nuestro modelo ("Nombre", "Variantes del nombre", "Género", "Géneros literarios que cultiva"; "Título", "Variantes del título", "Editorial”, "Lugar de edición", "Fecha de vencimiento de los 
derechos de autoría", "Número de control de la BNE"...), pero igualmente añaden otros nuevos de interés particular para su área, que pueden ser o no compartidos por otras colecciones: "Tipo de Colección", "Lenguas que traduce", "Exilio", "Geolocalización", etc. En este aspecto:

Mnemosine recoge unos contenidos muy avanzados, precisando $-y$ ofreciendo - por ello un modo completo y complejo de acceso a materiales a través de un modelo de búsqueda, de recuperación y de presentación pensado para la actividad académica. Aspectos de catalogación irrelevantes para el lector medio o el ámbito bibliotecario que pueden resultar de gran valía para el investigador son implementados en Mnemosine. Asimismo, las herramientas y funcionalidades que se ofrecen al usuario avanzan desde lo general hasta lo particular: desde aspectos como la búsqueda simple hasta la búsqueda cruzada o la anotación (Cotarelo Esteban, 2018: 27).

Definiendo una información común dentro unos mismos metadatos entre aquellos objetos virtuales que habrán de componer la colección, como campos $=>$ valor de las búsquedas cruzadas en la base de datos, la misma se generará de un modo automático. Dentro de la colección de obras de Literatura Infantil, por ejemplo, los resultados que aparecen directamente dentro de Mnemosine deben de provenir de discriminar la búsqueda a partir del dato "literatura infantil" dentro del campo o metadato "Materia" en las fichas bibliográficas de "obra" (objetos virtuales de clase obra) y solo en él, pues dentro del modelo de datos de fichas de "persona" (objetos virtuales de clase persona), puede aparecer esa misma información, empleando el mismo término, en autores en los que señalamos que han cultivado la literatura infantil dentro del campo "Géneros literarios que cultiva", pero que pueden tener o no obras de ese tipo dentro de Mnemosine. Tal dato, no obstante, podría servir para generar una colección biográfica de "Autores de Literatura Infantil en la Edad de Plata", o podrían cruzarse ambas informaciones para configurar a su vez otra colección. En la colección "Madrid en la Literatura" sucede algo semejante: al conformarla deben de aparecer los resultados provenientes de discriminar la búsqueda a partir del dato "Madrid" dentro del campo o metadato "Materia: lugar geográfico" en las fichas bibliográficas de "obra", y solo en él; sin contar, en cambio, si aparece en otro campo de la misma ficha como lugar de edición, etc., dato que podría constituir asimismo otro tipo de repertorio para otra colección. 
Y lo mismo podemos decir del resto de colecciones ya configuradas y de las potenciales futuras.

La forma en que se modelan los datos y en que se estructuran las colecciones responde a necesidades particulares. La colección "Autores en Exilio", por ejemplo, desarrollada por Lucía Cotarelo Esteban, ha sido modelada y estructurada para albergar una propuesta metodológica que tiene como fin rescatar y visibilizar un grupo concreto de escritores: aquellos que fueron víctimas del exilio político a causa de la Guerra Civil. Un subgrupo de esta colección, conformado por poetas que vivieron su exilio - o parte del mismo - en la costa Este norteamericana - base de estudio de la tesis doctoral de la coordinadora ${ }^{10}$ - , puede visualizarse al aplicarse el filtro "Exilio (País)": "Estados Unidos". En el modelo de datos diseñado para esta colección de carácter transdisciplinar, se han considerado para la ficha de "persona", junto a metadatos comunes ya a otras colecciones como "Forma desarrollada del nombre", "Variantes del nombre", "Nacionalidad" (fue frecuente el cambio de nacionalidad o la adopción de la ciudadanía del país de acogida), "Educación", "Actividad profesional", "Lenguas que escribe" (de gran interés para conocer la proyección que las lenguas peninsulares pudieron tener en el exilio), "Asociaciones a las que pertenece" (aspecto fundamental para los estudios migratorios y exiliares) o "Amistad con”, otros de naturaleza más específica como "Exilio", "Lugar de exilio" a nivel "País" y "Ciudad", "Muerte en exilio" y "Fecha de retorno a España"; y en la ficha bibliográfica de "obra", "Traducidos al", "Número de control de la Library of Congress" (que se suma al número de control de HathiTrust, de la BNE y OCLC ya existentes, pues permite apreciar la consideración y conocimiento por parte del país de recepción de esas obras escritas - aunque no siempre publicadas - en su territorio) junto a otros metadatos igualmente compartidos; $y$ en el apartado de Contenido, "Título del poema", "Geografía": "Latitud", "Longitud" y "Localización", elementos concernientes al plano de geolocalización (mapping, o cartografía digital), un aspecto aportado asimismo por esta colección y que funciona - por el momento - a dos niveles: a nivel de ficha de "persona", desde una perspectiva biográfica que permite visualizar

\footnotetext{
${ }^{10}$ Lucía Cotarelo Esteban, Poetas de La Otra Edad de Plata en el exilio estadounidense (costa Este): redes de socialización, biblioteca digital y geolocalización, defendida el pasado 3 de julio de 2019, en la Facultad de Filología de la UCM.
} 
su itinerario vital una vez fuera de España; y a nivel de "obra", con tres tipos de mapping distintos: de los espacios reales que las ha inspirado o donde ha sido redactada; de los espacios reales - convertidos en espacios poéticos - que pueden rastrearse en la propia obra; y de los espacios puramente poéticos, metafóricos (Cotarelo Esteban 2018: 32-37).

La colección "Literatura de Quiosco", organizada por Jeffrey Zamostny, profesor de literatura española de la Universidad de West Georgia, se sustenta en algunos metadatos de interés específico para este macrogénero rico en su temática, en su variedad estética e ideológica —aun dentro de la repetición de ciertas fórmulas consideradas comerciales-y muy prolífico en su número de títulos y ediciones: el género literario de las distintas colecciones - mayoritariamente de narrativa breve-, el lugar de publicación - por lo general grandes ciudades, donde surge el quiosco como construcción de urgencia para la venta de unas publicaciones cuyo consumo representa casi una normativa social-, el precio de venta o el número de ejemplares de una tirada, que difícilmente encontraremos en el catálogo de una biblioteca tradicional. Así, el diseño de los metadatos específicos de las colecciones de literatura de quiosco para la docencia y la investigación ha de implementar para la ficha de "obra" aspectos relacionados con la edición, como el título de la colección, director o responsable intelectual de la misma, número de títulos que contiene, de ejemplares de tirada, día de salida a la venta y periodicidad, puntos de venta, precio y características de formato; aspectos relacionados con el contenido como el subgénero, los personajes-tipo, los elementos de conflicto, estructuras, espacio y tiempo narrativos; aspectos relacionados con el público, como edades de los lectores, género del público, sociología del gusto - gusto encadenado al consumo, a un lector que demandaba narraciones en cuyas páginas pudiera reconocerse y se reflejasen tanto la sociedad que le había tocado vivir como sus ansias de renovación-, etc. (Romero, Bueren, Gayoso, 2017: 408-411).

De un modo muy similar, cada una con su genuina especificidad, se han ido configurando el resto de colecciones. Para generar la colección "Traductoras de la Edad de Plata", promovida por Amelia Sanz Cabrerizo, profesor titular de la UCM, se explicita la condición de "mujer" dentro del metadato de Género en la ficha de persona y asimismo el de "española" en la nacionalidad, excluyendo así — salvo alguna posible excepcióna determinadas autoras extranjeras que aparecen en Mnemosine en su condición de hispanistas traductoras de obras en la época, incorporadas 
como objeto virtual al considerarlas autoras secundarias; pero que no son al caso a la hora de conformar esta colección, al igual que en la de "Mujeres Intelectuales de la Edad de Plata". A continuación, si en las obras que tienen asignadas cada una de ellas como recursos, aparece el término "Traductor" dentro de la relación Persona-Obra que las une, se tratará de una autora a incorporar al elenco. En la ficha de "obra", por su parte, se definió — como señalamos con anterioridad - un nuevo recurso multievaluado, "reediciones", para establecer un estema o árbol jerárquico entre las primeras ediciones traducidas, publicadas en la Edad de Plata, y sus reediciones posteriores, que habían de estar dentro de la ficha de aquellas y no conformando fichas independientes, haciendo interminable la lista de recursos asociados a una autora. El modelo de metadatos de esta colección "incorporates elements that reflect the literary, historical and cultural characteristics of the collections 'specific metadata model for the women translators' collection", mostrando de ese modo cómo "these women also showed their interest in the writings of contemporary women by translating their works into Spanish or glossing foreign ideas about how the modern woman should be, think or behave. This digital collection shows the first steps of the intellectual women in the South of Europe" (Romero y Bueren, 2018).

La colección “Diálogos en la Edad de Plata”, a cargo de la especialista María Jesús Fraga, hace patente la especial floración que durante el primer tercio del siglo XX experimentó este género de larga estirpe literaria desde las antiguas culturas orientales y el mundo clásico grecolatino, con la etapa del humanismo renacentista del XVI como punto álgido. Al perfilar dentro de Mnemosine una colección de textos escritos en forma dialogada desde el metadato correspondiente de Género — con posibles subgéneros-, nuestra biblioteca viene a complementar la fructífera labor de catalogación y estudio que desde hace una década viene desarrollando al respecto el grupo de investigación UCM Dialogyca y su Biblioteca Digital de Diálogo Hispánico $(\mathrm{BDDH})^{11}$. Para la colección "Muertos en conflicto (1936-1939)", por su parte, se consideraron dentro del metadato Fecha de Fallecimiento de la ficha de "persona" todos aquellos autores "raros y olvidados" presentes en Mnemosine que murieron durante la Guerra Civil, conjunto especialmente numeroso — como cabía suponer-

${ }^{11}$ Dirección web: http://www.dialogycabddh.es/ [12/06/2019]. 
pero que no deja de impactar, sin embargo, una vez elaborado su listado y comprobadas las cifras totales, utilizando asimismo la herramienta Google Maps para localizar los lugares de defunción. A nivel metodológico, según los criterios temáticos y términos de la tragedia que se consideren podrá matizarse la presencia o no de determinados autores en cada caso; pero esto ya es cuestión de los intereses del especialista, la biblioteca responsable o el investigador ${ }^{12}$. Unos murieron directamente a causa de la guerra, en el frente de batalla o fusilados; otros no, pero como dijo Ortega y Gasset, tras la muerte de Miguel de Unamuno, el 31 de diciembre de 1936:

Ignoro todavía cuáles sean los datos médicos de su acabamiento; pero, sean los que fueren, estoy seguro de que ha muerto de "mal de España". [...] Ha inscrito su muerte individual en la muerte innumerable que es hoy la vida española. Ha hecho bien. Su trayectoria estaba cumplida. Han muerto en estos meses tantos compatriotas que los supervivientes sentimos como una extraña vergüenza de no habernos muerto también (2006: 409410).

En las colecciones individuales de autor como la de "Carmen de Burgos Colombine", aparecerán todas las obras que tenga ese autor/a en Mnemosine. Pero para ir finalizando este recorrido en las colecciones temático-literarias de Mnemosine, nos detendremos en una de las más particulares: la colección "Protociencia-ficción Agustín Jaureguízar", compuesta por títulos de este característico subgénero pertenecientes a la biblioteca privada del mencionado coleccionista, la más completa existente en España sobre esta especialidad, que han sido digitalizados por la biblioteca de la UCM. Para formar esta colección no se importaron datos de manera masiva; la estudiosa Marta Correa Román se encargó de modelar los metadatos e introducir manualmente los datos. El problema, sin embargo, radica en que muchas de las obras escaneadas tienen derechos de autor y la biblioteca de la Complutense no permite colgarlas de su web ${ }^{13}$.

\footnotetext{
${ }^{12}$ De hecho, en 2016, la BNE en su sección Escritores en la BNE, creó la subcolección "Escritores fallecidos en 1936", con el catálogo y las semblanzas de algunos de los autores fallecidos en el primer año de guerra, bajo el asesoramiento de José-Carlos Mainer; además de trabajar, con la llegada del año 2017, en la selección y digitalización de los autores que fallecieron durante aquel trágico año y que ya puede ser difundida libremente, en virtud de la Ley de Propiedad Intelectual. ${ }^{13}$ Para el acceso a las obras, véase http://eprints.ucm.es/view/coleccion/protocienciaficcion.html y http://repositorios.fdi.ucm.es/mnemosine/colecciones.php [12/06/2019]. La diferencia de cantidad de títulos entre un repositorio y otro es de 53 frente a 124, respectivamente.
} 
A esta situación debería de encontrarse, de cara al futuro, una solución satisfactoria y legal. Una posibilidad sería colgar los libros en una nube y que a través de un enlace dentro de Mnemosine se localizaran allí, pudiendo acceder a ellas mediante clave encriptada y teniendo control de que los textos solo se van a utilizar con fines de investigación. Esta circunstancia, en fin, que revela la necesidad de que haya más textos en abierto, nos conduce a uno de los principales puntos de análisis del siguiente epígrafe sobre perspectivas y conclusiones.

\section{PERSPECTIVAS Y CONCLUSIONES}

Mnemosine, Biblioteca Digital de La Otra Edad de Plata pretende ser “... a field of international experimentation for the creation of interoperable semantic networks through which a large group of scholars could generate innovative research and theoretical reading models for literary texts" (Romero y Bueren, 2018). Mnemosine es completamente reconfigurable - en el plano de contenido, de metadatos, etc.—y, a diferencia de otras bibliotecas digitales, está pensada, diseñada y enriquecida desde y para la investigación; un laboratorio electrónico para crear datos, acceder a textos, generar repertorios y reutilizar los contenidos de otros portales, superando las limitaciones de los bibliotecarios a la hora de catalogar pues estos catalogan para que un usuario encuentre una obra y él en el depósito, pero no considera otros aspectos que no necesita para su cometido, al no ser investigador ${ }^{14}$.

A través de la herramienta Clavy, la importación de datos desde grandes bibliotecas y repositorios (HathiTrust, $B D H \ldots$ ) constituye la primera fase en la generación de datos de Mnemosine, a la que siguen la fase de adaptación, limpieza y enriquecimiento de esos datos. A Clavy

\footnotetext{
${ }^{14}$ Una dicotomía que las actuales bibliotecas digitales van difuminando cada vez más, como afirma Díez Carrera (2012, 37-38): "Existe cierta sintonía entre las voces centro de documentación y biblioteca especializada, y cada vez tiene menos sentido hablar de la distinción entre bibliotecario y documentalista [...] Hoy, sin embargo, bibliotecarios y documentalistas manejan por igual las tecnologías en un mundo inmerso en ellas, tienen un conocimiento y unas técnicas comunes, como lo son los retos e inquietudes profesionales. En un mundo digital y global, las bibliotecas y centros de documentación aspiran a tener documentos digitales y digitalizados, todo tipo de documentos; a describir el conjunto y sus partes, y de la manera más amplia posible, tanto en lo formal como en lo conceptual a través de una gran variedad de metadatos para su posterior recuperación; a crear diferentes productos...; tareas en definitiva similares".
} 
se integrará @Note, una aplicación que permite cargar libros electrónicos y efectuar actividades de anotado de un modo colaborativo, desarrollada igualmente por el grupo de investigación ILSA. En las bibliotecas digitales actuales, hay muy pocas funciones que nos permitan trabajar texto, anotar o copiar; de ahí la utilidad de implementar herramientas digitales (toolkits) para llevar a cabo investigación e interdisciplinariedad, como anotación colaborativa, líneas de tiempo, mapas conceptuales, geolocalización con Google Maps, etc. La incorporación del motor de anotado@Note en Clavy añade así al modelo la posibilidad de anotar los recursos, los textos deseados para nuestras fichas ampliando la versatilidad de la herramienta en los procesos de investigación o docencia.

Un modelo de datos específico como el de Mnemosine permitirá la búsqueda simple y avanzada de datos cruzados que dará lugar a una recuperación de la información más selectiva, mejor dirigida. Si — por ejemplo- se asocian los metadatos "Título de la colección" y "Obras" saldrá un listado de obras asociadas a cada una de las colecciones; si les asociamos otras informaciones como "Responsable", "Fechas" o "Periodicidad", la casuística podría dar lugar a datos muy interesantes desde el punto de la sociología literaria (Romero, Bueren y Gayoso, 2017: 411412). Pensar con la lógica de los datos enlazados facilita la comprensión del modelo; si en Mnemosine se distinguen dos tipos principales de entidades: autores y obras, en el momento en que se adopte el modelo de datos enlazados, este esquema se sustituirá por la red de elementos y relaciones que subyacen al mismo. La búsqueda de datos enlazados puede crear diversidad de lecturas que sacarían a la luz diferentes temas de carácter docente e investigador; si se cuenta con un modelo complejo, bien definido, con registros minuciosamente descritos con datos de calidad, las posibilidades de explotación de la información son tantas como podamos imaginar.

El principal objetivo de una biblioteca de "raros y olvidados" de la Edad de Plata como Mnemosine es replantear determinados principios tradicionales para demostrar las posibilidades de reconstrucción y relectura, a través de recursos digitales, de la historiografía literaria superando determinadas categorías canónicas en las que se ha estructurado tradicionalmente la producción literaria de la Edad de Plata. Para ello, tener acceso al texto es fundamental en nuestra biblioteca. A la hora de 
digitalizar, la vigente ley de propiedad intelectual de $2014^{15}$ continúa siendo igual de rígida: 80 años desde la muerte del autor. Solo en el caso de las obras "huérfanas" permite una mayor agilidad a la hora de digitalizar. La ley de depósito legal obliga también a registrar publicaciones en línea, objetos virtuales y contenidos en general. Así, el fondo digitalizado en la Complutense abarca fundamentalmente libro antiguo (hasta 1870); desde EE.UU., HathiTrust está procurando poner en abierto obras de autores que ellos han digitalizado, al encontrarse en fondos de sus bibliotecas universitarias ${ }^{16}$; que no tengan copyright según la legislación española; la Biblioteca Nacional de España publica anualmente un listado de nuevos autores que pasan a dominio público al cumplirse sus derechos: por lógica, con el transcurso de los años cada vez son más los escritores de la Edad de Plata que se hayan en esta circunstancia - a día de hoy, todos los fallecidos antes de 1939-. La BNE no puede difundir libremente - como es evidente- contenidos que no cumplan la normativa del copyright, pero ya en 2015 llevó a cabo, de acuerdo con Mnemosine, una nueva digitalización de fondos literarios de la Edad de Plata ("raros y olvidados") que no estaban incluidos cuando se realizó la primera importación. También, en estos últimos años, a través de la open library Internet Archive BookReader (dominio archive.org [12/06/2019]) hemos podido añadir nuevos libros digitalizados en abierto en nuestra base de datos, incorporando enlaces externos a este cada vez más valioso portal.

Algunas cifras de lo conseguido hasta ahora y de lo que constituye Mnemosine en la actualidad, nos hablan de 1.067 autores totales (192 mujeres autoras, 126 ilustradores, 63 compositores, 51 mujeres traductoras...) y 4.029 obras totales (1.050 con enlace a su lectura en abierto). Tras una primera financiación al proyecto de tres años a cargo del MEC, una ayuda concedida a continuación por la Fundación BBVA al proyecto de investigación "Modelo unificado de Gestión de Colecciones Digitales con Estructuras Reconfigurables: Aplicación a la Creación de Bibliotecas Digitales Especializadas para Investigación y Docencia” (Ref. HUM14_251) del grupo ILSA dirigido por José Luis Sierra Rodríguez,

\footnotetext{
${ }^{15}$ Ley 21/2014, de 4 de noviembre (véase https://www.boe.es/boe/dias/2014/11/05/pdfs/ BOE-A-2014-11404.pdf [12/06/2019]).

${ }^{16}$ Las cifras actuales de HathiTrust hablan de un número de volúmenes digitalizados que sobrepasa los 16 millones, de los que más de seis están en dominio público (véase https://biblioteca.ucm.es/ hathitrust [12/06/2019]).
} 
permitió durante 2015 y 2016 el desarrollo de la herramientas informáticas que sustentan a Mnemosine, como Clavy y otras externas como la de geolocalización o la de anotación. El proyecto "Edición Literaria Electrónica" (eLITE-CM) (Ref. H215/HUm-34-26) con financiación del Fondo Social Europeo y encabezado por la profesora titular de la UCM María Goicoechea de Jorge, en el que participó LOEP junto con otros grupos de investigación de la misma Universidad, nos permitió fundar entre 2016 y 2018 una serie de colecciones de libros interactivos (La Mujer Moderna en la Edad de Plata, Colección Infantil, Madrid en la Literatura de la Edad de Plata) que, editadas mediante el programa Madgazine de edición digital y alojadas dentro de la página web de la $\mathrm{BNE}^{17}$, sucesivamente hemos ido incorporando al repertorio de Mnemosine, dando el salto desde el libro digitalizado al digital ${ }^{18}$. Hoy día, en 2019 , un proyecto de $\mathrm{I}+\mathrm{D}+\mathrm{i}$ del MEC recién concedido, titulado "Mnemosine: hacia la historia digital de La Otra Edad de Plata — producción, almacenamiento, uso y difusión(MNEMOSINE-2020)", nos permitirá seguir avanzando en investigación y en difundir nuestros resultados, afrontando nuevos retos para el futuro: nuevos autores, nuevas colecciones, nuevas fuentes de datos (datos.bne. es, VIAF, DBpedia, Wikipedia ${ }^{19}$, Hispana, Internet Digital Library of Free Books, Europeana...), modelos de datos específicos que partan de modelos de datos existentes (FRBR, FOAF) para asegurar la interoperabilidad, crear subcolecciones dentro de una misma colección (mediante etiquetado, búsqueda afinada, aplicación automática de filtros...), ordenación de las obras enlazadas como recursos dentro de cada autor con un criterio cronológico antes que alfabético (más científico pero también más lento y laborioso si no hay un motor de búsqueda automático), mejorar la eficacia

\footnotetext{
${ }^{17}$ Véase http://www.bne.es/es/Colecciones/LibrosInteractivos/Subcolecciones/edad-platainteractivo.html [12/06/2019].

${ }^{18}$ El libro digitalizado "es un libro que se ha pensado y diseñado para papel y que luego ha sido convertido a algún formato digital. En este caso, el libro no tiene ningún elemento extra, tan solo es el texto y las ilustraciones pertinentes [...] Frente a los libros digitalizados, los digitales han sido ideados y diseñados teniendo en cuenta todas las posibilidades y las funciones de los libros electrónicos y los dispositivos de lectura, y proporcionan una nueva experiencia lectora [...] para aprovechar todas las posibilidades de este entorno: texto, imagen, videos e hiperenlaces, lo que marca una diferencia considerable que va más allá del soporte" (Gómez, García, Cordón, Arévalo, 2016: 68-69).

${ }^{19}$ A través de la web sobre cómo descargar datos desde Wikipedia podrían completarse metadatos de Mnemosine. https://m.wikidata.org/wiki/Wikidata:Data_access/es [12/06/2019].
} 
de buscador y filtros para que las consultas tengan el éxito esperado, crear material didáctico multimedia sobre "raros y olvidados" de la Edad de Plata, que los usuarios puedan crear sus propias colecciones importando datos directamente de las grandes bibliotecas, plantear la posibilidad de crear colecciones restringidas (Google Drive permite que los enlaces a recursos puedan ser seguidos únicamente por ciertos usuarios) para superar en parte las limitaciones impuestas por los derechos de autor, conseguir que Clavy sea cada vez más intuitivo para los usuarios (el proceso de manipulación del modelo para limpiar los datos de MARC21 tras una importación masiva es un proceso complejo que requiere conocimiento previo) y que se pueda descargar y usar como herramienta para la investigación...

El proceso queda abierto, por tanto, en el desarrollo técnico y de contenidos de nuestra biblioteca digital y la apertura de nuevas colecciones. Pero siempre bajo una clara premisa: sin el conocimiento certero y directo, sin la quietud de una lectura profunda, atenta y analítica, de las obras los nuevos entornos digitales no nos conducirán a ninguna parte. Es historia literaria interpretativa lo que aspiramos a promover, a partir de una nueva accesibilidad en los datos y textos. La información bibliográfica, autores y libros, contenida en Mnemosine es la materia prima fundamental que nosotros proporcionamos a los investigadores, además de las herramientas necesarias para generar entidades y relaciones específicas de la Edad de Plata, colecciones literarias y, en definitiva, investigación.

\section{AGRADECIMIENTOS}

El autor desea hacer constar su agradecimiento a José Luis Bueren Gómez-Acebo, actual director técnico de la BNE después de haber dirigido su Biblioteca Digital Hispánica y Sistemas de Información entre 2014 y 2017; a Joaquín Gayoso Cabada, miembro del Departamento de Ingeniería del Software e Inteligencia Artificial (ISIA) y del grupo de investigación ILSA de la Facultad de Informática de la UCM; a Laura Sánchez Gómez, gestora cultural e investigadora, miembro del grupo de investigación LEETHI (Literaturas Españolas y Europeas del Texto al Hipermedia) y cofundadora de Ciberia Project, plataforma de investigación sobre edición y creación digital; y a Dolores Romero López, profesora titular de Literatura de la Facultad de Filología de la UCM, miembro del Departamento de Literaturas Hispánicas y Bibliografía, directora del grupo de investigación 
"La Otra Edad de Plata: proyección cultural y legado digital" (LOEP) y creadora y coordinadora de Mnemosine, Biblioteca Digital de la Otra Edad de Plata (1898-1936), las muchas horas de reuniones, trabajo cooperativo y mutuo aprendizaje en la gestación y desarrollo de esta apasionante empresa, así como a todos los compañeros e investigadores que han colaborado - y colaboran - activamente en la misma.

\section{REFERENCIAS BIBLIOGRÁFICAS}

CEBALLOS VIRO, A. ed. (2014). La retaguardia literaria en España (1900-1936). Madrid: Visor Libros.

CODINA, L1. y Rivera, C. (2006). "La web semántica”. En Tendencias en documentación digital, J. Tramullas (coord.), 9-54. Gijón: Trea. COTARELO ESTEBAN, L. (2018). "La colección 'Poetas en el exilio' en Mnemosine: Biblioteca Digital de La Otra Edad de Plata". En Entornos digitales. Humanidades y Ciencias Sociales en la Universidad Complutense de Madrid, D. Romero López y M. Joaquín Salamanca López (coords.), 25-39. Ciudad de México: Red de Humanidades Digitales.

DÍEZ CARRERA, C. (2012). La biblioteca digital. Gijón: Trea.

ENA BORDONADA, A. ed., (2013). La Otra Edad de Plata. Temas, géneros y creadores (1898-1936). Madrid: Editorial Complutense. FERNÁNDEZ-PAMPILLÓN, A.; GAYOSO, J.; SARASA, A. y SIERRA, J. L. (2018). "De OdA a Clavy, dos aplicaciones orientadas a la creación de repositorios de objetos digitales académicos". En Entornos digitales: Humanidades y Ciencias Sociales en la Universidad Complutense de Madrid, D. Romero López y M. Joaquín Salamanca López (coord.), 61-83. Ciudad de México: Red de Humanidades Digitales.

GARCÍA CAMARERO, E. y GARCÍA MELERO, L. A. (2001). La biblioteca digital. Madrid: Arco / Libros.

GÓMEZ DÍAZ, R.; GARCÍA RODRÍGUEZ, A.; CORDÓN GARCÍA, J. A. y ALONSO ARÉVALO, J. (2016). Leyendo entre pantallas. Gijón: Trea.

GONZÁLEZ SORIANO, J. M. (2017). Luis Bello, cronista de la Edad de Plata (1872-1935). Salamanca: Diputación. 
ORTEGA Y GASSET, J. (2006). Obras completas. Tomo V (1932-1940). Madrid: Taurus.

ROMERO LÓPEZ, D. (2014). "Hacia la Smartlibrary: Mnemosine, una biblioteca digital de textos literarios raros y olvidados de la Edad de Plata (1868-1936). Fase I". Humanidades Digitales: desafios, logros y perspectivas de futuro. Janus, Anexo 1, 411-422. Disponible en https://www.janusdigital.es/anexos/contribucion. htm? id =37 [12/06/2019].

ROMERO LÓPEZ, D.; BUEREN GÓMEZ-ACEBO, J. L. y GAYOSO CABADA, J. (2017). "Modelling Kiosk Literary Collectios for the Mnemosyne Digital Library". En Kiosk Literature of Silver Age Spain: Modernity and Mass Culture, J. Zamostny y S. Larson (eds.), 399-417. Bristol, UK / Chicago: Intellect.

ROMERO LÓPEZ, D. y BUEREN GÓMEZ-ACEBO, J. L. (2018). "Networking Women Translators in Spain (1868-1936) and their Presence in the Mnemosyne Digital Library". The Electronic Library 36.2, 305-318. Disponible en línea: https://doi.org/10.1108/ EL-02-2017-0026 [12/06/2019].

SÁINZ DE ROBLES, F. C. (1971). Raros y olvidados (la promoción de "El Cuento Semanal"). Madrid: Prensa Española.

ZHANG, A. B. (2008). Creating Digital Collections. A Practical Guide. Oxford: Chandos.

Recibido el 13 de marzo de 2020.

Aceptado el 29 de abril de 2020. 